Questions de communication

\title{
Intégrer les politiques aux divertissements
}

Résistances, coopération et concessions de l'univers politique

Involving Politicians in Entertainment Programmes. Oppositions, Cooperation

and Concessions of the Political Sphere

\section{Pierre Leroux et Philippe Riutort}

\section{(2) OpenEdition}

12 Journals

Édition électronique

URL : http://journals.openedition.org/questionsdecommunication/8646

DOI : 10.4000/questionsdecommunication.8646

ISSN : 2259-8901

Éditeur

Presses universitaires de Lorraine

Édition imprimée

Date de publication : 31 décembre 2013

Pagination : 19-35

ISBN : 978-2-8143-0182-5

ISSN : 1633-5961

\section{Référence électronique}

Pierre Leroux et Philippe Riutort, «Intégrer les politiques aux divertissements », Questions de communication [En ligne], 24 | 2013, mis en ligne le 02 février 2016, consulté le 30 avril 2019. URL

http://journals.openedition.org/questionsdecommunication/8646; DOI : 10.4000/ questionsdecommunication.8646 
PIERRE LEROUX

Centre de recherche sur l'action politique en Europe

Institut d'études politiques de Rennes

Université catholique de l'Ouest/L'Université Nantes Angers Le Mans

F-49000

pierre.leroux@uco.fr

PHILIPPE RIUTORT

Groupe d'analyse politique

Université Paris Ouest Nanterre La Défense

F-92000

riutortp@yahoo.fr

\title{
INTÉGRER LES POLITIQUES AUX DIVERTISSEMENTS. RÉSISTANCES, COOPÉRATION ET CONCESSIONS DE L'UNIVERS POLITIQUE
}

\begin{abstract}
Résumé. - La participation régulière de personnalités politiques à des émissions de divertissement a débuté en France à la fin des années 90. L'invitation des politiques à des mises en scène hors des routines des émissions politiques repose sur des logiques d'intérêts qui sont analysées en mettant en relation les dispositifs des émissions et les caractéristiques de la population de professionnels reçus à différentes périodes. Ce bilan montre la nécessité d'une analyse fine des positions au sein de l'univers politique pour comprendre les résistances, les coopérations et les concessions qui ont marqué la courte histoire de la légitimation de ces nouvelles scènes de la politique, tandis que, à l'issue de l'élection présidentielle française de 2012, la participation de l'ensemble des acteurs politiques aux divertissements semble acquise et ce type d'émissions légitimées.
\end{abstract}

Mots clés. - Télévision, politique, talk shows, discours politique, divertissement, dispositif. 
L

a participation du personnel politique aux émissions de divertissement relève-t-elle d'une simple logique d'invitation émanant des professionnels de la télévision? En d'autres termes, la réception d'une personnalité politique répond-elle à une simple demande à laquelle les politiques, avides de mise en spectacle, d'exhibition, de cultiver ou d'accroitre leur notoriété et de réaliser des performances d'audience, s'empresseraient de répondre ? Un tel propos rejoint sans doute la vision commune et largement propagée du métier politique qui, en raison de l'omniprésence des discours médiatiques consacrés aux stratégies de communication politique, conduit à appréhender la conquête des mandats et le maintien à des positions de pouvoir comme la construction et l'entretien d'un « charisme » reposant principalement sur la présence médiatique et sur son instrumentalisation. La fin (électorale) justifiant les moyens (médiatiques), « toutes [les] formes de communication sont considérées, par les candidats et leurs conseillers, comme les composantes d'une stratégie multi-médias au sein de laquelle la télévision joue le rôle de navire-amiral » (Cayrol, Mercier, 2002 : 6). En France, le succès rapide des émissions de divertissement accueillant régulièrement des invités politiques, puis leur légitimation comme tribune politique accrédite, en apparence, la thèse selon laquelle les promoteurs de ces émissions partageraient avec le personnel politique le souhait de s'adresser au public le plus large et, si possible, d'enregistrer l'audience la plus importante, quelles que soient les modalités de cadrage à l'œuvre (empruntant au registre classique de l'émission politique ou volontairement en rupture avec elle). Si parler politique (si l'on veut bien prendre le terme dans son sens le plus large) peut représenter un intérêt pour les animateurs, ces professionnels de l'audiovisuel désormais consacrés (Leroux, Riutort, 2006)' enrichissent leurs registres de prise de parole et entretiennent des relations de proximité avec des responsables politiques, ce qui constitue une assurance non négligeable face aux aléas de carrières structurellement instables, voire une revanche symbolique sur le groupe des journalistes politiques qui les a longtemps méprisés. Mais, l'activité politique et ses logiques de champ (positions et intérêts relatifs à celles-ci) ne sauraient mettre l'ensemble des agents politiques face aux mêmes contraintes et attentes à l'égard des médias, ni face aux mêmes enjeux lors des différents moments d'une carrière politique. Cette prise en compte des positions et des capitaux détenus par les personnalités politiques permet préalablement de rompre avec deux illusions. La première est celle d'une attente indifférenciée d'apparitions médiatiques équivalentes quelles que soient les tribunes offertes et les conditions d'apparitions. En un mot, il n'est pas sûr que les politiques sans exception « cherchent [l']audimat désespérément », selon le titre d'un ouvrage (Malherbe, 2007). La seconde est celle d'une compréhension du recrutement des émissions politiques qui pourrait ne pas tenir compte des capitaux

' En 2005, le président de la République, Jacques Chirac, a eu recours à trois animateurs (et à un seul journaliste) pour présenter un débat « avec les jeunes » avant le référendum sur la ratification du traité établissant une constitution pour l'Europe. 
politiques des participants (Le Foulgoc, 20 I0) et surtout de la valeur mouvante de ceux-ci. En outre, ces émissions s'alignent de plus en plus nettement sur le rythme sans cesse croissant de l'actualité politique et médiatique qu'elles construisent en même temps qu'elles la répercutent.

La participation des politiques aux divertissements se présente donc sous des aspects contrastés, comme nous le montrons à travers l'étude du recrutement des invités politiques de ces émissions que nous analyserons en premier lieu sous deux aspects : un aspect synchronique, prenant en compte l'état de l'offre d'émissions et de « scènes » (Balandier, 1992) de représentation de la politique à un moment donné et repérant d'éventuelles différences entre les émissions, un aspect diachronique visant à enregistrer d'éventuelles évolutions (générales ou relatives à une même émission). Nous expliquerons ces évolutions en analysant, d'une part, le poids de certaines variables au sein de la population accueillie par les émissions conversationnelles et, d'autre part, les dynamiques sous-jacentes ayant conduit, dès la fin des années 2000, à la participation quasi généralisée de l'ensemble du personnel politique de premier plan.

\section{Convaincre les politiques}

La facilité apparente avec laquelle les premières émissions parviennent à s'assurer la présence régulière des responsables politiques tendrait à prouver que, dès l'origine, elles répondaient à des attentes ou des besoins de l'univers politique. Pourtant, si l'on examine dans le détail la capacité des trois émissions conversationnelles pionnières, on remarque que la présence des politiques n'est pas allée de soi. Présentateur et producteur du Vrai Journal (Canal +, 1996-2006)², Karl Zéro est le premier à intégrer dans son émission l'invitation hebdomadaire d'une personnalité politique. II semble réussir son pari puisque, les deux premières années, seules cinq personnalités extérieures au cercle des professionnels de la politique (sur 30 en première saison et 39 en deuxième) sont présentes. Une première partie des invités correspond clairement à l'objectif de l'animateur, même s'il ne s'agit pas des représentants les plus légitimes (Lhérault, 2002), puisque ces invités politiques occupent une place relativement marginale au sein de leur propre formation ${ }^{3}$. Un autre type d'invités vient compléter ce recrutement. II s'agit de participants dotés d'une certaine notoriété auprès du public, mais ne détenant pas de mandat ${ }^{4}$, représentants

\footnotetext{
2 Le Vrai Journal était diffusé le dimanche à l'heure du déjeuner.

3 Michel Charasse, Michel Rocard ou BernardTapie (les trois premiers invités, tous affiliés au Parti socialiste - PS) sont dans ce cas, de même que - à des degrés divers - Philippe de Villiers (Mouvement pour la France - MPF), Jean-Claude Gaudin (Rassemblement pour la République - RPR), Bernard Kouchner (PS), Roland Dumas (PS), Jean-Pierre Chevènement (PS) ou Éric Raoult (RPR), invités la première année.

${ }^{4}$ Des personnalités comme Jean-Edern Hallier, écrivain polémiste au comportement souvent provocateur lors de ses apparitions à la télévision (quatrième invité), Pierrette Le Pen, ex-femme du
} 
de la société du spectacle plus que de la société civile. Leur propension au scandale, aux révélations ou aux provocations constitue un atout susceptible de compenser leur faible poids politique. Mais, l'ambition de l'animateur est bien d'avoir accès à des représentants de premier plan et ces invités ne constituent qu'un pis-aller palliant sa difficulté à mobiliser des personnalités de premier plan. D'ailleurs, ces invités disparaitront progressivement par la suite, en tant que personnes (ils ne seront plus reçus), mais surtout en tant que profil d'invités. Largement inspirée de l'entretien journalistique classique et finalement limitée à quelques audaces formelles ${ }^{5}$, la formule permet de s'assurer de la présence de personnalités elles aussi marginales, mais dont les prises de position paraissent davantage respectables. Qu'il s'agisse de l'évêque Jacques Gaillot en conflit avec la hiérarchie catholique et, en conséquence, fortement médiatisé pour ses prises de position publiques, ou de Danielle Mitterrand épouse de l'ex-président de la République et militante (invitée la première saison et reçue à nouveau par la suite), ces invités bénéficient d'une réputation moins tapageuse et d'une parole plus mesurée, mais, en contrepartie, incarnent une faible dimension spectaculaire. Les premières personnalités prestigieuses et proprement politiques n'acceptent l'invitation que dans un deuxième temps, dynamique déclenchant par un effet d'entraînement la participation de détenteurs de positions de plus en plus élevées. Le rythme hebdomadaire de l'émission conduit la production à construire un lien constant avec l'univers politique par la collaboration de deux salariés introduits dans l'univers politique - un « à gauche » et l'autre « à droite », visiblement « très bien rémunérés » selon les mots du producteur de l'émission Michel Malaussena (entretien, nov. 20ll) - pour s'assurer la présence « du plus connu au bon moment » (idem), les relations personnelles de l'animateur ne permettant pas à elles seules d'alimenter l'émission. Dès la troisième saison, les innovations de l'émission se sont en grande partie banalisées et l'institutionnalisation est confirmée par la participation de certains ténors de la politique ${ }^{6}$. Ensuite, deux évolutions notables se font jour. Le nombre des invités extérieurs à l'univers politique augmente (pour atteindre le taux approximatif d'un quart des invités), faisant une large place à l'univers du spectacle, et le cercle des politiques invités se referme en partie à travers la sollicitation des mêmes individus, tout en étant globalement constitué de détenteurs de postes

leader de l'extrême droite ayant livré aux médias des détails de sa vie avec son ex-mari, ou Christine Deviers-Joncour (invitée à deux reprises) « ex-maitresse » du ministre Roland Dumas impliquée dans l'affaire Elf (affaire politico-financière ayant éclaté en 1994 et mis au jour un important réseau de corruption au sein de cette entreprise majoritairement contrôlée par l'État) et confiant les détails de sa relation dans des livres et de nombreux médias.

5 Parmi les quelques audaces formelles dont fait preuve Le Vrai Journal, on relève notamment: l'interpellation par le prénom et le tutoiement de l'invité, une fois son autorisation obtenue devant la caméra ; un questionnement apparemment « sans concession » (selon l'animateur) à travers l'utilisation d'un langage direct ; l'interpellation familière et directe, probablement accentuée par le montage, et le déroulement de cette séquence en public.

${ }_{6}^{6}$ Parmi les personnalités politiques de premier plan présentes dans la troisième saison du Vrai Journal, on compte Pierre Mauroy, Henri Emmanuelli, Nicolas Sarkozy, Simone Veil, Lionel Jospin, Alain Juppé et François Hollande. 
appartenant au premier cercle de la vie politique (ministres, chefs de partis, notamment) ${ }^{7}$. Certaines personnalités (essentiellement politiques) invitées à de multiples reprises constituent progressivement un cercle privilégié soulignant de nouvelles limites à l'espace des invitations ${ }^{8}$ : une partie du personnel politique résiste toujours aux sollicitations de l'émission et cette dernière évite les individus potentiellement peu adaptés au dispositif et aux règles spectaculaires tout en favorisant ceux qui s'y prêtent.

Si, du fait de sa position de pionnier et de sa forte consommation d'invités, Le Vrai Journal donne une vision assez précise de l'état de la relation champ politique/télévision, les deux divertissements créés ensuite occupent des positions sensiblement différentes. Vivement dimanche (France 2, 1998-) ${ }^{9}$ et Tout le monde en parle (France 2, 1998-2006) avancent des ambitions à la fois plus modestes (par le nombre d'invités politiques sollicités) et plus hautes (les invités s'intègrent à un dispositif plus contraignant, ou pour le moins inusité à l'égard des politiques). Pour Michel Drucker, il s'agit d'appliquer une formule de célébration habituellement réservée aux invités du show business ; pour Thierry Ardisson, il s'agit d'intégrer les politiques à un talk show programmé à un horaire tardif (ce qui, au regard des conventions de l'époque sur le service public autorise à questionner l'intimité de ses invités).

Rétrospectivement, la réussite de Vivement dimanche paraît exemplaire. L'émission ne présente apparemment aucun risque pour les invités politiques car elle fonctionne sur des valeurs de convivialité, de complicité avec l'équipe de chroniqueurs et sur la valorisation explicite de la personnalité de l'invité. À la longue, elle s'est révélée comme un rendez-vous fréquenté par le personnel politique de premier plan (Premier ministre en exercice, ministres et principaux leaders politiques) d'une façon comparable à celle des émissions politiques traditionnelles les plus prestigieuses. Les invités sont des personnalités ayant accumulé un crédit politique et médiatique d'une importance dépassant souvent celle du mandat détenu au moment de l'invitation. Cependant, il serait erroné de considérer que ces critères proprement politiques (comme la position occupée) dominent strictement les principes de sélection des

\footnotetext{
7 Parmi les personnalités politiques de premier plan invitées plusieurs fois, on compte notamment Nicolas Sarkozy, Ségolène Royal, Simone Veil, Lionel Jospin, Alain Juppé, François Hollande. L'émission offre aussi un large éventail de représentants des organisations partisanes (à l'exclusion de l'extrême droite) : l'extrême gauche, avec Lutte ouvrière et la Ligue communiste révolutionnaire (dès la première saison), le Parti communiste, les diverses tendances écologistes (Brice Lalonde, Corinne Lepage, puis Les Verts en deuxième saison avec Daniel Cohn-Bendit, Dominique Voynet et Noël Mamère), pour des raisons qui tiennent moins à des volontés politiques particulières qu'à la disponibilité de représentants peu sollicités par la télévision.

${ }^{8}$ Sur les cinq premières saisons du Vrai Journal, 195 individus sont invités, dont 41 (21 \%) à plusieurs reprises. Totalisant I 28 invitations, ces individus sollicités plusieurs fois constituent à eux seuls $65 \%$ du total. La moyenne de leur présence est de 3,12 fois, ce qui souligne une clôture sur lui-même du cercle des invités.

9 Vivement dimanche est diffusée le dimanche après-midi
} 
invités. Pour fonctionner pleinement, le dispositif de l'émission doit, mutatis mutandis, transposer les principes de sélection en vigueur au sein du monde du spectacle : ancienneté de la carrière, traits consensuel de l'image publique passant par la détention de la respectabilité (l'hommage des concurrents dans la famille du show business comme dans celle de la politique est là pour en témoigner) et de fidélité (qui peut tenir à certaines constances apparentes dans les choix et les prises de positions). Potentiellement, l'émission n'a donc pas pour vivier le champ professionnel de la politique, mais celui plus réduit des entrepreneurs politiques consacrés par le temps et la reconnaissance des pairs dont le profil se prête à l'angle d'approche de l'émission. Ainsi, contre une vision simpliste qui ferait de l'émission une valorisation standardisée de ses invités allant de soi pour les producteurs ou les participants, faut-il souligner que l'effort de construction conjoint de mise en récit du parcours, de formatage de la représentation de soi et d'emprunt à des registres variés de conversation ne saurait s'adresser à des invités politiques débutants ou émergents. Bien sûr, dans les premiers temps, la production doit composer avec les résistances et les refus de certains invités politiques, mais le profil de ses premiers invités n'en correspond pas moins au projet initial et au dispositif de l'émission. Parmi ceux qui voulaient bien accepter d'inaugurer cette nouvelle formule, les deux premiers invités en 1999 (Daniel Cohn-Bendit et Arlette Laguiller) sont éloignés du premier cercle du pouvoir, et ce n'est qu'avec la troisième invitée (Martine Aubry, numéro deux du gouvernement au poste de ministre de l'Emploi et de la Solidarité), que le vivier des invités s'élargit : selon l'animateur, il s'agissait alors d'une première prenant valeur de test auprès de l'univers politique et qui permit d'amorcer le recrutement en faisant appel, en premier lieu, aux ressources de son carnet d'adresses (Drucker, 200I). Par son recrutement parmi les détenteurs de positions politiques dominantes, Vivement dimanche pourrait donc être comparée aux émissions politiques au statut le plus élevé, mais c'est surtout la rareté des places disponibles qui conduit à la sélectivitél0. L'émission doit s'abstenir de recevoir des invités politiques en période d'élection car le traitement hagiographique des personnalités l'assimile largement à une entreprise de communication politique, mais elle n'est pas assez directement politique (ne serait-ce qu'en raison du faible nombre d'invités politiques) pour assumer - ce que d'autres émissions de divertissement feront ensuite (à la façon du Grand Journal ou d'On n'est pas couché) - la dimension représentative de la politique en période électorale en adoptant un strict dispositif égalitaire. D'ailleurs, pour les membres du premier cercle politique, elle apparaît vite comme la seule émission de divertissement envisageable dans l'offre existante à l'époque (les deux autres émissions étant Le Vrai Journal sur Canal + et Tout le

10 Par exemple, pendant les années 2005-2006, seuls cinq professionnels de la politique seront invités sur le plateau de Vivement dimanche. Aux frontières de la politique, et de façon cohérente avec le projet d'ensemble de l'émission, les épouses de l'ancien président de la République (Danielle Mitterrand, 08/01/06) et de celui en exercice (Bernadette Chirac, janv. 2007) sont reçues. 
monde en parle sur France 2) '!. Cette sélectivité autorise la production à faire un choix par rapport à la demande de participation. Les préférences accordées à certains types d'invité et les modalités de mise en scène participent d'une redéfinition de la représentation télévisuelle de la politique.

\section{Les dispositifs sous tension}

Le cas de Tout le monde en parle ${ }^{12}$ (France 2), présentée par Thierry Ardisson, est proche sur le principe et sensiblement différent dans la forme. Après le succès d'audience mitigé d'une première version (autour de « débats de société » thématiques) ${ }^{13}$, l'émission va s'affirmer comme un divertissement culturel à partir du début de l'année 1999 en posant les fondements d'une formule conversationnelle largement imitée par la suite. L'invitation de politiques (19 invités politiques en 1997, 20 en 1998) prend souvent comme prétexte la sortie d'un livre. L'émission de Thierry Ardisson semble avoir plus difficilement que ses concurrents (Le Vrai Journal, Vivement dimanche) accès à l'univers politique, et ne peut disposer d'invités liés à l'actualité si ce n'est de façon biaisée (notamment pour la promotion d'un livre) ${ }^{14}$. La présence des politiques, catégories très minoritaire ( $4 \%$ des invités), ne se concrétise qu'à travers le recours à des élus de second plan - pour moitié des députés et des sénateurs, en l'absence quasi totale de membres du gouvernement (Amey, Leroux, 2012 : 7-20). Si donc, pour la plus grande partie des politiques, la participation à l'émission représente un coût trop élevé (notamment en raison de l'image alors controversée de l'animateur et du privilège accordé à la déstabilisation des invités par le montage), l'émission n'en inaugure pas moins un type de dispositif et un mode de relation à la politique qui fera école dans la première moitié des années 2000 avec la multiplication des émissions conversationnelles organisées sur un principe de tension avec les invités politiques (Leroux, Riutort, 2012). Tandis que le principe de déstabilisation des invités politiques (en particulier grâce à l'instauration d'un mélange des genres qui les place en minorité) est érigée en règle ${ }^{15}$, la pénétration de l'univers politique ne se réalise encore que très partiellement.

\footnotetext{
"Jean-Pierre Raffarin, Premier ministre, ne participe qu'à cette seule émission de divertissement lorsqu'il occupe cette fonction, alors qu'il prend par ailleurs une position publique hostile à l'égard des propositions novatrices de mise en scène du personnel politique. En 2003, il interdit aux ministres de son gouvernement de participer à une émission de télé-réalité prévue sur TFI et pour laquelle Jean-François Copé (2006 : 55-57) avait été sollicité.

12 Tout le monde en parle est diffusée de 1998 à 2006 les samedis en deuxième partie de soirée.

13 Selon T. Ardisson (2005: 331), « l'audience est une déception hebdomadaire qui se répète », l'émission a failli être déprogrammée et a été contrainte de changer de formule.

14 Par exemple, les multiples invitations de Christine Deviers-Joncour durant l'affaire Elf témoignent du lien biaisé à l'actualité.

15 De ce point de vue, les talk shows à la française ne semblent en rien déroger aux nombreuses expériences étrangères où les invités politiques sont les seuls à subir un traitement défavorable de la part des animateurs (Schütz, 1995).
} 
Tableau I. Le capital des invités des émissions conversationnelles (200 I-2006)16.

\begin{tabular}{|l|r|l|}
\hline Capital politique & $\begin{array}{l}\text { Nombre } \\
\text { d'invitations }\end{array}$ & $\begin{array}{l}\text { Taux d'invitations } \\
\text { par rapport au nombre } \\
\text { total d'invités politiques } \\
\text { (en \%)' }\end{array}$ \\
\hline $\begin{array}{l}\text { Présidents de la République } \\
\text { et Premiers ministres }\end{array}$ & 0 & \\
\hline $\begin{array}{l}\text { Ministres d'État } \\
\text { et principaux ministres }\end{array}$ & 3 & 2,4 \\
\hline $\begin{array}{l}\text { Ministres délégués } \\
\text { et secrétaires d'État }\end{array}$ & 4 & 3,2 \\
\hline Anciens ministres & 53 & 42,7 \\
\hline Chefs de partis & 14 & 11,2 \\
\hline Députés/sénateurs & 62 & 50 \\
\hline $\begin{array}{l}\text { Maires, conseillers municipaux, } \\
\text { généraux et régionaux }\end{array}$ & 16 & 12,9 \\
\hline Non-élus & 23 & 18,5 \\
\hline $\begin{array}{l}\text { Divers (hommes politiques } \\
\text { étrangers, diplomates, etc.) }\end{array}$ & 16 & 12,9 \\
\hline Total (pour I24 individus invités) & 191 & \\
\hline
\end{tabular}

La prépondérance des invités politiques au sein des émissions conversationnelles relève du « second marché » (Leroux, Teillet, 2006), c'est-à-dire d'un ensemble hétérogène de détenteurs de positions de second plan dans l'espace politique, mais qui, à divers titres, en l'absence de participation des acteurs politique de premier plan, représentent un compromis acceptable pour les producteurs des émissions (visibilité, lien avec l'actualité, dispositions à s'intégrer à ce type de dispositifs). Leur relative faiblesse à l'égard des médias (ils ne sont pas en position d'être invités dans les émissions politiques classiques les plus prestigieuses) s'accorde avec des formes d'expertise ou de responsabilités qui les autorisent à une parole plus libre (que, par exemple, celle d'un ministre en exercice). Pour une faible part, le personnel politique visible dans les émissions conversationnelles comprend les entrants du champ politique (invités en grande partie pour l'originalité de leur positon : le benjamin de l'Assemblée, une députée ordinaire qui exposerait la vérité du terrain), mais surtout des anciens titulaires de positions élevées (anciens ministres) dotés d'une forte notoriété mais souvent

\footnotetext{
16 Pour les tableaux I et 2, trois émissions conversationnelles obéissant à des logiques spectaculaires proches (en dépit de différences non exposées ici) ont été retenues : Tout le monde en parle de janvier 200 I à mai 2006 ; On ne peut pas plaire à tout le monde (France 3, le dimanche en prime time) de septembre 2004 à mai 2006 ; 20 h 10 pétantes (Canal +, le samedi et le dimanche durant le journal télévisé) de septembre 2004 à mai 2006. II s'agit de trois talk shows hebdomadaires recevant plusieurs invités sur leur plateau, où aucun invité ne peut se prévaloir d'un statut d'invité unique ou privilégié.

17 Le total dépasse $100 \%$ car on a choisi d'attirer l'attention sur les titres plus que sur leurs porteurs : ainsi les anciens ministres sont-ils, par exemple, systématiquement comptabilisés (et donc souvent pris en compte deux fois) quelle que soit, par ailleurs, leur fonction au moment de l'invitation (député, par exemple).
} 
écartés des émissions politiques. Ainsi trouvent-ils des tribunes pour un discours qui s'accorde avec le traitement de la politique par ces émissions (par exemple, lors de la parution d'ouvrages à vocation dénonciatrice). La place significative et a priori surprenante occupée par les députés ordinaires se comprend mieux lorsque l'on considère leurs autres attributs ${ }^{18}$ et leurs dispositions médiatiques. Ainsi le tribun communiste s'insurgeant contre l'appareil, l'ancien présentateur du journal télévisé se portant candidat à l'élection présidentielle et le conseiller régional membre d'un parti conservateur rendant publique son homosexualité apparaissent-ils comme des invités de choix de ces programmes. La présence considérable des élus locaux (tableau I, 12,9\%), incarnant les territoires ou jouant le rôle de témoin sur une question emblématique, sert à donner une vision moins professionnalisée de la politique : disposés à mettre en évidence leur faiblesse relative de maîtrise du métier politique, ils témoignent d'une certaine proximité avec leurs électeurs... qui les éloignerait des professionnels de la politique. Le nombre élevé de non-élus parmi les invités (tableau I, 18,5\%) est un signe d'incontestable affranchissement à l'égard du champ politique qui fait écho au souci permanent de promotion de la société civile (si l'on veut bien prendre ce terme dans son acception médiatique et politique) émanant des animateurs-producteurs : du philosophe ou du savant devenus ministres à l'ancien militant humanitaire reconverti, ces invités-là possèdent, en outre, quasiment tous, à un titre ou à un autre, un lien avec l'univers des médias (éditorialiste, collaborateur d'un magazine ou d'une émission de télévision, voire liens familiaux et matrimoniaux).

Tableau 2. Les invités par affiliation politique ${ }^{19}$.

\begin{tabular}{|l|r|l|}
\hline Affiliations politiques & Nombre & $\begin{array}{l}\text { Taux en rapport } \\
\text { avec le nombre total } \\
\text { d'invités politiques (en \%) }\end{array}$ \\
\hline Extrême droite (FN) & 1 & 0,08 \\
\hline Droite : UMP + UDF (dont UDF) & $41(2)$ & 33 \\
\hline Gauche : PS + PC (dont PC) & $45(7)$ & 36,2 \\
\hline Les Verts & 9 & 7,2 \\
\hline Extrême gauche (NPA, LO) & 12 & 9,6 \\
\hline Divers & 16 & 12,9 \\
\hline Total & 124 & 100 \\
\hline
\end{tabular}

18 Député durant la période étudiée, Jack Lang détient le record d'invitations du corpus, certainement en raison de son capital politique, mais également en raison de son insertion ancienne au sein des univers du spectacle et de la communication.

${ }^{19}$ Liste des abréviations utilisées : FN (Front national) ; LO (Lutte ouvrière) ;NPA (Nouveau Parti anticapitaliste); PC (Parti communiste) ; PS (Parti socialiste) ; UDF (Union pour la démocratie française) ; UMP (Union du mouvement populaire). 
Il est également nécessaire de se livrer à une lecture de la représentativité partisane (voir tableau 2) en intégrant l'emprise de la logique spectaculaire de ces émissions. Si un certain équilibre politique est respecté entre les deux grands blocs du champ politique (la gauche et la droite du gouvernement) - en dépit de notables exceptions ${ }^{20}$ - la règle journalistique de représentativité des courants politiques - que suivent les émissions politiques classiques - peut être contournée : le refus explicite d'inviter des représentants de l'extrême droite en témoigne ${ }^{21}$. De même, la surreprésentation de l'extrême gauche est notable ${ }^{22}$ : marginalisée dans les émissions politiques classiques, elle se trouve ici créditée d'une utile capacité à cliver les débats.

On peut constater que pendant la première moitié des années 2000 les émissions conversationnelles ont largement ouvert leur porte aux invités politiques et que, en ce sens, elles ont élargi la représentation de l'univers politique. Parfois porté à leur crédit sur le plan politique, cet élargissement ne procède pourtant en grande partie que des limites induites par les dispositifs. Comme le montre le succès et la pérennité des deux émissions pionnières, la participation des politiques du premier rang est conditionnée de leur part à une évaluation de type coût/bénéfice : ces deux émissions offrent des garanties d'une prise de risque potentiellement faible (absence d'agressivité, thématiques prévisibles, séquences sans enjeux), et éventuellement des bénéfices escomptées (consécration pour Vivement dimanche, rajeunissement d'image pour Le Vrai Journal). Quoique ces deux modèles ne puissent être dupliqués à l'infini, en raison du lien de la formule de l'émission avec la personnalité de son animateur qui lui permet de se démarquer des modèles existants (l'émission de variété et le magazine de célébration des célébrités pour Vivement dimanche, l'interview politique classique pour Le Vrai Journal) et de s'insérer dans une logique de programmation (le public du dimanche dans les deux cas avec des objectifs d'audience limités pour Le Vrai Journal). Par leur formule même, « les trois cruels talk-shows parisianopeoplesque » (Anizon, 2002 : 59) ${ }^{23}$ construits sur le modèle de tension dans la relation avec les invités politiques, de déstabilisation, de raillerie voire d'humiliation définissent des limites dans l'accès aux responsables politiques, d'autant que ces

\footnotetext{
${ }^{20}$ On a pu remarquer la surreprésentation des invités politiques classés à gauche dans Tout le monde en parle (Robin, 2006). Ce phénomène pourrait être conjoncturel :il semble plus risqué pour un représentant de la majorité de défendre la politique du gouvernement dans ce type d'émissions qui, en revanche, se prête assez bien à recevoir les critiques. Mais on doit aussi tenir compte du fait qu'une grande partie des interlocuteurs classés dans la catégorie « divers », sans revendiquer d'étiquettes partisanes et de partis pris politiques, pourraient être facilement rangés du côté des personnalités conservatrices.

${ }^{21}$ Le seul invité du corpus est, à ce moment, le porte-parole du Mouvement pour la France (MPF) de Philippe de Villiers, Guillaume Peltier, ancien membre du Front national (FN).

22 Dans le corpus utilisé, l'extrême gauche est représentée par Arlette Laguiller (Lutte ouvrière - LO), Olivier Besancenot (Ligue communiste révolutionnaire - LCR) et José Bové (syndicat Confédération paysanne).

${ }^{23}$ Emmanuel Anizon fait alors référence à Tout le monde en parle, On ne peut pas plaire à tout le monde et, « dans une moindre mesure [à] On a tout essayé ». Le Grand Journal sur Canal + reprendra globalement le même principe, mais en évoluant progressivement vers une préservation relative de la parole politique.
} 
émissions s'enrichissent de séquences allant toujours plus loin dans ce sens ${ }^{24}$. Les difficultés à atteindre les représentants les plus prestigieux de l'univers politique sont le reflet des contraintes fortes d'un genre qui veut que le politique consente à abandonner potentiellement toute espérance de bénéfice politique direct au profit d'intérêts faibles, marginaux et somme toute hypothétiques, pour le personnel politique dominant. La notoriété résultant de tout passage à la télévision, l'élargissement espéré du public, la démonstration possible de qualités spectaculaires (humour, convivialité, sens de la répartie) n'ont sans doute - hors du temps de campagne électorale - pas un intérêt suffisamment fort pour mobiliser le personnel politique aux affaires.

\section{Une refondation du divertissement politique}

La rentrée 2006 est marquée par la transformation globale du paysage des émissions conversationnelles avec la disparition de plusieurs émissions ${ }^{25}$. Le resserrement de l'offre autour de trois émissions phares sur les grandes chaînes s'instaure à travers des formules moins ouvertement transgressives à l'égard de la politique et plus nettement en prise avec l'actualité. Ainsi le passage deThierry Ardisson de France 2 à Canal + (et le lancement de Salut les Terriens) se fait-il au prix d'un aseptisation relative du dispositif, explicitement imposée par la chaine $^{26}$, le dispositif du Grand Journal sur Canal +, concomitante de la disparition de $20 \mathrm{~h} 10$ pétantes, ne reprend pas les rubriques mettant explicitement en cause les invités et réserve un temps aux politiques en début d'émission, les confrontant à un journaliste politique ${ }^{27}$, à l'écart des personnalités du spectacle, tout comme On n'est pas couché qui fait aussi appel à deux intervieweurs spécialisés. Cette concession à l'univers politique permet très rapidement un accès élargi au premier cercle de la vie politique (principaux membres du gouvernement, chefs de partis...). En moins de trois saisons, On n'est pas couché reçoit 80 invités politiques (contre I 19 en dix ans pour Tout le monde en parle). Tandis que Thierry

\footnotetext{
${ }^{24}$ Par exemple, on citera certaines déclinaisons des « interviews formatées » de Th. Ardisson, une parodie de psychanalyse de l'invité et la rubrique « Chez maman », ou la chronique de Stéphane Guillon dans 20 h 10 pétantes, les petites rubriques et séquences comme « La minute blonde » ou « La boîte à question » dans Le Grand Journal qui mettent en cause explicitement les invités politiques.

25 Parmi les émissions déprogrammées en 2006 figurent Tout le monde en parle (France 2), T'empêche tout le monde de dormir (M6) qui avait pris la suite d'On ne peut pas plaire à tout le monde (France 3), Vendredi et samedi pétantes et Le Vrai Journal (Canal +).

26 L'aseptisation du dispositif repris par Salut les Terriens se traduit notamment par la disparition de l'humoriste Laurent Baffie, que sa fonction de sniper (chargé sur le plateau des réparties cinglantes) avait rendu célèbre (entretien avec Stéphane Simon, directeur de Télé Paris - société productrice de l'émission -, novembre 20 I I).

27 À la rentrée 20 I I, la séparation, de la rubrique « Le petit journal » du Grand Journal, se fait au prix d'un artifice : la lecture humoristique de l'actualité politique, ainsi reléguée vers la fin de l'émission, peut se réaliser en présence d'un invité politique en plateau, mais sur un mode complice avec celuici et qui n'est souvent pas sans rappeler l'humour sans conséquence des chansonniers d'autrefois.
} 
Ardisson n'a reçu pour la première fois un ministre en exercice qu'en troisième saison (Jean Glavany, ministre de l'Agriculture), Laurent Ruquier accueille l'un des principaux ministres (Gilles de Robien, ministre de l'Éducation nationale) et cinq autres membres du gouvernement dès la première saison.

Tableau 3. Les invités politiques d'On n'est pas couché, 2006-201228.

\begin{tabular}{|l|r|r|}
\hline Fonctions & $\begin{array}{l}\text { Nombre } \\
\text { d'invités }\end{array}$ & $\begin{array}{l}\text { Proportion par rapport } \\
\text { au nombre total } \\
\text { d'invités politiques } \\
\text { (en \%) }\end{array}$ \\
\hline Présidents et Premiers ministres & 0 & 0 \\
\hline Ministres d'État et principaux ministres & 13 & 8 \\
\hline Ministres délégués et secrétaires d'État & 30 & 18,5 \\
\hline Anciens ministres & 14 & 8,5 \\
\hline Leaders et porte-parole de partis & 35 & 21,5 \\
\hline Députés/sénateurs & 54 & 33 \\
\hline Élus locaux & 9 & 5,5 \\
\hline Non-élus & 8 & 5 \\
\hline Total & 163 & 100 \\
\hline
\end{tabular}

Tableau 4. Les invités politiques de Salut les terriens, 2006-20I $2^{29}$.

\begin{tabular}{|l|r|r|}
\hline Fonctions & $\begin{array}{l}\text { Nombre } \\
\text { d'invités }\end{array}$ & $\begin{array}{l}\text { Proportion par rapport } \\
\text { au nombre total } \\
\text { d'invités politiques } \\
\text { (en \%) }\end{array}$ \\
\hline Président et Premier ministre & 0 & 0 \\
\hline Ministre d'Etat et principaux ministres & 13 & 7,2 \\
\hline Ministres délégués et secrétaires d'Etat & 25 & 14,4 \\
\hline Anciens ministres & 19 & 12 \\
\hline Leaders et porte-parole de partis & 29 & 35 \\
\hline Députés/sénateurs & 69 & 20,4 \\
\hline Élus locaux & 14 & 4,8 \\
\hline Non élus & 5 & 6 \\
\hline Total pour 223 émissions & 174 & 100 \\
\hline
\end{tabular}

Les transformations du Grand Journal sont encore plus notables : sur une saison moyenne (mars 2008-mars 2009), ce ne sont pas moins de 42 ministres et secrétaires d'État, 4 I membres de l'opposition, 13 ex-ministres et ex-chefs de partis, ainsi que 20 autres personnalités politiques - soit environ « une personnalité

${ }_{28}$ La présence massive des leaders et porte-parole de partis est due en grande partie à la saison 2011 2012 précédant l'élection présidentielle. Cette remarque est également valable pour le tableau suivant.

29 Sur la période 2006-2012, I74 invités politiques ont été reçus sur le plateau sur un total de 223 émissions (soit une présence dans 78 \% des émissions). 
politique pour 10 premières parties d'émissions » selon Patrick Amey (2009) ${ }^{30}$. Aujourd'hui, l'émission a réussi à accueillir sur son plateau, jour après jour, tous les acteurs politiques de premier plan, à partir du poids qu'elle entend donner aux derniers rebondissements médiatiques de la politique.

L'explication tautologique qui voudrait qu'un (re)formatage autour de l'infotainment soit à l'origine de ce nouveau rapport au politique confond la cause et l'effet. C'est précisément parce que les émissions ont changé qu'elles peuvent prétendre instaurer un rapport avec l'actualité politique avec des modalités adaptées à leur projet (celle de l'infotainment, si l'on accepte le flou qui entoure cette dénomination). La réorientation du projet éditorial n'a été rendu possible qu'au prix de concessions importantes aux politiques : une séparation (au moins symbolique) avec l'univers du show business (qui était auparavant l'ingrédient principal du mélange des genres), la disparition de séquences attentatoires aux personnalités politiques, l'apparition ou le renforcement du rôle des spécialistes et la potentialité de tenir un discours plus explicitement politique en rapport avec l'actualité du moment font partie des principales transformations des dispositifs ${ }^{31}$.

Lorsqu'elle prend la peine de saisir dans la durée les parcours médiatiques des professionnels de la politique, l'analyse statistique des invitations politiques permet de tirer deux conclusions principales. Toutes les invitations télévisées ne sauraient être équivalentes dans une carrière politique (ce que tend à gommer l'agrégation des invitations pour une personnalité) et, même si désormais les émissions de divertissement sont pleinement intégrées à un dispositif de communication d'un responsable politique ${ }^{32}$, une présence dans une émission de divertissement ne saurait remplacer une invitation en tant qu'invité principal dans une émission politique de premier plan - le face-à-face avec des journalistes ou des opposants restant toujours plus légitime que la participation à un divertissement. L'emploi des catégories « divertissement » et «politique »- qui ne valent que par l'opposition qu'elles consacrent - tant par les acteurs que par les chercheurs,

\footnotetext{
${ }^{30}$ Voir les statistiques établies par P. Amey dans le présent dossier de Questions de communication. L'Express (17/02/09) note ainsi l'élévation du niveau des invité reçus : « Sur les 25 premiers numéros de la saison 2008-2009, Michel Denisot en avait reçu 15 ! Parmi eux, les trois ennemis de Reims (Aubry, Delanoë, Royal), deux anciens Premiers ministres (Raffarin et Villepin), les deux "B" anti Sarko (Bayrou et Besancenot), quatre ministres et une première dame ».

${ }^{31}$ Ainsi l'interview des politiques se déroule-t-elle dans une première partie dans Le Grand Journal, et les interactions avec les autres invités sont limitées dans On n'est pas couché (le temps de l'entretien, l'invité quitte la table commune pour s'installer à part dans un fauteuil), tout comme dans Salut les Terriens (et à la différence de Tout le monde en parle dont elle a pris la suite). L'entretien avec des spécialistes est valorisé (avec Jean-Michel Aphatie et Bruno Donnet pour Le Grand Journal, les duos d'intervieweurs d'On n'est pas couché), mettant en partie en retrait les aspects les plus visible du spectacle (les politiques ne participent plus à des séquences humoristiques), tout comme celles qui les raillaient directement (« La minute blonde » dans Le Grand Journal, les interviews formatées de T. Ardisson) n'existent plus. Enfin, l'absence des snipers s'amusant au dépend des invités politiques, ingrédient notable des divertissements de la période précédente, a contribué à transformer en profondeur la relation aux invités politiques.

32 Sur ce point, voir les développements dans P. Leroux et P. Riutort (2013).
} 
tend à occulter, lorsqu'elles servent de pierre angulaire au classement statistique, tant les rapprochements non négligeables et croissants observables entre les deux genres de programmes précités (Leroux, Riutort, 2012) que l'apparition de tout un ensemble de programmes invitant des responsables politiques qui empruntent aussi bien au « politique » qu'au « divertissement », sans pour autant pouvoir être confondu avec eux.

En effet, les transformations des dispositifs des programmes télévisuels incluant des invités politiques conduisent à l'adoption croissante de formes mixtes alliant, au sein de la même émission, l'alternance de séquences au cours desquelles priment alternativement le sérieux ou la légèreté. Ces évolutions devraient inciter à refuser une fois pour toutes d'envisager l'opposition entre les émissions de divertissement - appréhendées comme un ensemble homogène - et les émissions politiques, qui font également l'objet d'un traitement indifférencié au nom du sérieux qui leur est prêté mais rarement examiné plus avant ${ }^{33}$. Au contraire, il semble que l'analyse de nouveaux dispositifs de prise de parole télévisée gagnerait à tenir compte de la multiplication des registres d'expression publique qui pourraient être interrogés à partir de la trajectoire politique de l'invité dont l'exposition médiatique est en grande partie redevable.

\section{Dépasser l'opposition entre "sérieux» et « divertissement»}

L'invitation télévisée ne saurait procéder de la seule volonté de médiatisation émanant du politique ou de la fantaisie des médias dans la mesure où elle découle, la plupart du temps, de la position institutionnelle du politique qui tend à l'instituer en un invité difficilement évitable.

L'exemple de Manuel Valls - successivement ou conjointement, député « rénovateur » du Parti socialiste (PS), chargé des questions de sécurité au PS, député-maire d'une ville de banlieue parisienne, allié de Ségolène Royal dans sa tentative de prise du contrôle du PS, candidat à la primaire socialiste, porteparole du candidat François Hollande à l'élection présidentielle -, illustre bien le fait qu'une personnalité politique fait l'objet de séquences (variables) de médiatisation fortement tributaires de sa position occupée dans le champ politique. Si Manuel Valls peut faire figure de responsable politique émergent, doté de caractéristiques originales jusqu'au milieu des années 2000, notamment en raison de certaines prises de position savamment en décalage avec la direction du parti auquel il appartient, et qui s'avèrent bénéfiques, en retour, en termes de visibilité médiatique, celle-ci croît fortement lorsque ses positions s'affirment au sein du champ politique. On peut aussi relever que la visibilité

33 Sur le sérieux prêté aux émissions politiques mais rarement examiné plus avant, voir P. Leroux et P. Riutort (2013, surtout pp. 195-196). 
médiatique de Manuel Valls progresse en rapport, tant dans les émissions de divertissement que dans les émissions politiques, à la fin de la période, qui méritent ainsi d'être appréhendées, conjointement et simultanément, comme autant d'opportunités offertes à un moment donné à un professionnel de la politique souhaitant s'exposer médiatiquement et non comme des dispositifs qui seraient a priori exclusifs et étrangers les uns aux autres. En outre, en pratique, la prise en compte de la diversité et de la multiplication récente des programmes susceptibles d'accueillir des invités politiques conduit le chercheur confronté à la taxinomie des programmes télévisés à devoir accroître le nombre des catégories d'émissions, sauf à aplanir tous les obstacles qui se présentent à lui.

Graphique I. Invitations de Manuel Valls sur les plateaux télévisés ${ }^{34}$.

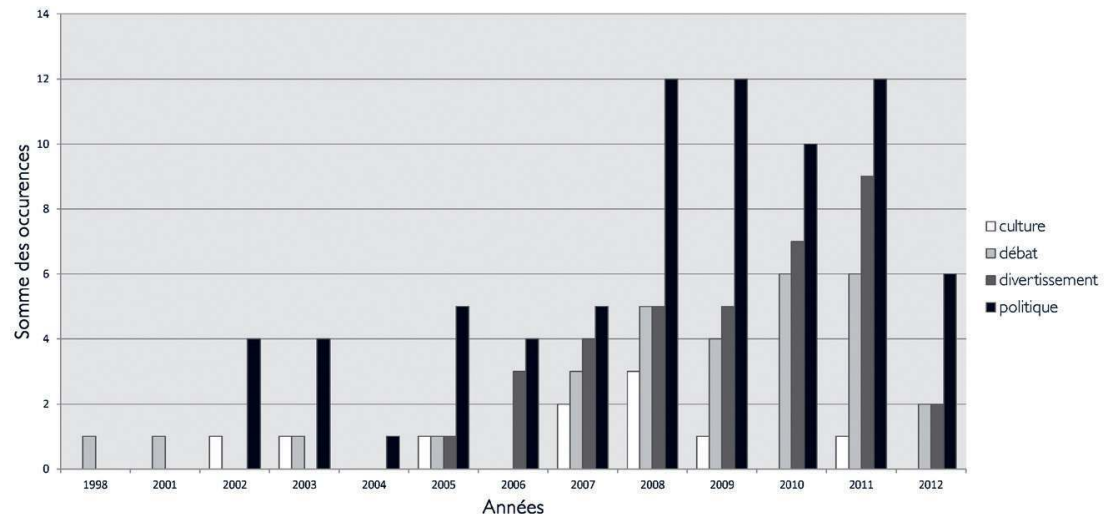

Ainsi se sont imposées d'autres catégories, à côté de « politique » (émissions politiques relevant de la direction de l'information et présentées par des journalistes) et de « divertissement » (émissions relevant de la direction des programmes et présentées par des animateurs dans une case réservée), comme ${ }^{35}$ 《 débat » ou « culture ». La multiplication des émissions de plateaux entrecoupées de reportages (comme Zone Interdite ou Capital sur M6, Revue et

\footnotetext{
${ }^{34}$ Les invitations politiques de Manuel Valls en plateau ont été reconstituées à partir des données de I'Institut national de l'audiovisuel (Ina) que nous remercions pour leur aide efficace. Les catégories retenues ont été construites empiriquement à partir de la diversité des émissions de plateau. II n'a pas été possible de reprendre à notre compte les catégories de l'Ina qui ne classent pas les émissions selon des dispositifs et des formats de prises de parole, mais, la plupart du temps, selon des genres d'émission, genres qui s'avèrent, en pratique, fortement hybrides. Nous avons ensuite retenu quatre genres (classification évidemment évolutive et contestable) « politique », 《 divertissement », « débat », « culture », dans la mesure où les deux principales catégories habituellement retenues (« politique » et « divertissement ») nous ont paru excessivement restrictives.

35 Nous ne tenons pas spécifiquement aux catégories retenues pour elles-mêmes, puisqu'elles pourraient être remaniées au fur et à mesure de l'évolution des dispositifs des émissions. Elles constituent simplement un indicateur des transformations des dispositifs qui tendent à rendre désormais quasiment impossible un classement binaire.
} 
Corrigé sur France 5) au cours desquelles des invités, parfois politiques, sont conviés à réagir constitue bien un genre d'interventions difficilement classable dans le cadre des « émissions politiques » ou du « divertissement » et qui s'apparentent davantage au « débat ». De même, l'expansion d'émissions « culturelles » (Chez FOG sur France 5, Le Bateau livre sur France 5, Semaine critique sur France 2) où les invités politiques sont reçus pour présenter un livre et participer à un débat avec le journaliste et/ou l'animateur, ou encore celle d'émissions de plateau aux thématiques empruntées aux magazines ( $C$ à vous, France 5 ) relèvent d'un dispositif de prise de parole encore différent des précédents où les tonalités sérieuses et de légères alternent d'une séquence à l'autre de l'émission et où certains propos politiques peuvent côtoyer des échanges portant sur des sujets variés, de l'exposition de ses goûts littéraires à l'intervention à brûle-pourpoint sur un sujet d'actualité ${ }^{36}$. II semble qu'une gradation s'opère entre le sérieux et le léger au sein même des différents programmes, d'une séquence à l'autre, conduisant à instaurer des différences de degrés bien plus que de nature entre ce qui relèverait aujourd'hui du « divertissement » et de la « politique ».

\section{Conclusion}

Au terme d'un cycle de transformation des émissions conversationnelles qui a duré moins d'une quinzaine d'années, l'accès de celles-ci à l'univers politique dans son ensemble peut être interprété comme une victoire des animateurs sur leurs invités politiques, cependant au prix de concessions non négligeables faites aux membres de l'univers politique. Si les professionnels du divertissement n'ont pas réalisé leur projet initial de désacralisation du politique visant à l'annexer au divertissement, ils sont parvenus à imposer, dans les nouveaux dispositifs, des formes de cadrage du traitement de la politique largement distinctes de ce qu'elles étaient dans les émissions politiques classiques, lors de l'apparition des premières émissions conversationnelles. Face aux détenteurs des plus hautes positions au sein de l'univers politique, les gages donnés (comme la présence de journaliste spécialisés et la disparition des séquences pièges) paraissent insignifiants au regard du prestige et de la distinction apportés par la présence des invités politiques de premier plan, d'autant que ces transformations se sont opérées - pour l'instant - sans mettre en péril l'audience des émissions. La monstration de la politique mise en spectacle télévisuel s'effectue en partie au profit des metteurs en scène, des animateurs-producteurs et de leurs co-animateurs, revanche symbolique indéniable puisque l'accès aux puissants ne leur est plus interdit et qu'ils disposent, en outre, du pouvoir consistant à les rendre visibles auprès du plus large public.

36 L'émission culturelle Semaine critique (France 3, 2010-201 I) adjoint au ton sérieux de l'émission un des dispositifs du divertissement en intégrant la séquence de l'humoriste Nicolas Bedos invité, en fin d'émission, à indirectement « tirer le portrait » de certains des invités présents sur le plateau. 


\section{Références}

Amey P., 2009, La parole à la télévision. Les dispositifs des talk-shows, Paris, Éd. L'Harmattan.

— 20I2, « La politique dans Le Grand Journal. Investissement parodique, registre critique », pp. 107-124, in : Amey P., Leroux P., dirs, L'échange politique à la télévision. Interviews, débats, et divertissements politiques, Paris, Éd. L'Harmattan.

Amey P., Leroux P., dirs, 20I2, L'échange politique à la télévision. Interviews, débats, et divertissements politiques, Paris, Éd. L'Harmattan.

Anizon E., 2002, « Les hommes politiques dans les émissions de divertissement », Les dossiers de l'audiovisuel, 102, pp. 59-60.

Ardisson T., 2005, Confession d'un baby-boomer, Paris, Flammarion.

Balandier G., 1992, Le pouvoir sur scènes, Paris, Balland.

Cayrol R., Mercier A., 2002, «Télévision politique et élection », Les dossiers de l'audiovisuel, 102, pp. 6-7.

Copé J.-F., 2006, Promis, j'arrête la langue de bois, Paris, Hachette.

Drucker M., 200 I, « J'ai fait sortir les politiques de leur ghetto », Le Monde télévision, I6- 17 déc., pp. 2-3.

Malherbe A. de, 2007, Politiques cherchent audimat désespérément, Paris, A. Michel.

Le Foulgoc A., 2007, Les représentations politiques reconfigurées par le divertissement à la télévision française. Des programmes pris dans des stratégies et des temporalités, thèse en science de l'information et de la communication, Université Panthéon-Assas.

- 2010, Politique et télévision, Paris, Ina Éd.

Leroux P., Riutort P., 2006, « La consécration de l'animateur. Appréciation d'un métier et affirmation d'une position : les métamorphoses de Thierry Ardisson », Réseaux, 139, pp. $219-248$.

— 2012 « La parole politique à la télévision. Les dynamiques d'émergence de nouveaux régimes discursifs », pp. 4I-58, in : Amey P., Leroux P., dirs, L'échange politique à la télévision. Interviews, débats, et divertissements politique, Paris, Éd. L'Harmattan.

— 2013, La politique sur un plateau, Paris, Presses universitaires de France.

Leroux P., Teillet P., 2006, « Second marché médiatique et carrière politique. L'exemple de Roselyne Bachelot », pp. 439-456, in : Cohen A., Lacroix B., Riutort P., dirs, Les formes de l'activité politique. Éléments d'analyse sociologique. XVIII- $-X X^{e}$ siècles, Paris, Presses universitaires de France.

Lhérault M., 2002, Le vrai journal décrypté. Chez Karl Zéro, l'information comme nulle part ailleurs, Paris, Éd. Nouveau Monde.

Robin J., 2006, Ils ont tué la télé publique, Paris, Éd. du Journalisme continu.

Schütz A., « Entertainers Experts or public Servants ? Politicians'Self-Presentation on Television Talk Shows », Political Communication, 2 (I2), 1995, pp. 2 I I-221. 
